\title{
Um Estudo Exploratório sobre a Alfabetização Computacional em São Paulo
}

\section{Alternative Title: An Exploratory Study on Computer Literacy in São Paulo}

\author{
Edmir Parada Vasques Prado \\ Universidade de São Paulo - EACH \\ Av. Arlindo Bettio, 1000 - Ermelino Matarazzo \\ CEP: 03828-000 São Paulo/SP \\ eprado@usp.br
}

\author{
Marco Alberto Wang \\ Universidade de São Paulo - EACH \\ Rua Alexandre Dumas 2220, 60 andar \\ CEP 04717-917 São Paulo/SP \\ marco.wang@usp.br
}

\begin{abstract}
RESUMO
Ao longo do tempo, tem-se presenciado o aumento da disseminação dos dispositivos computacionais na sociedade. Entretanto, pesquisas recentes evidenciam situações de indivíduos munidos de dispositivos de TI, mas despreparados tecnologicamente. Dado este contexto, evidencia-se que há uma aprendizagem mínima necessária sobre aspectos computacionais, os quais atribui-se o termo Alfabetização Computacional (AC). O objetivo deste estudo exploratório é avaliar a relevância dos aspectos da AC na formação da base de conhecimentos dos jovens brasileiros. Este trabalho caracteriza-se por ser uma pesquisa exploratória que utiliza a técnica de entrevistas em profundidade para captar percepções de três instituições brasileiras relacionadas ao tema de estudo. A pesquisa confirma a relevância dos conhecimentos e habilidades da AC relatados na literatura e identifica os aspectos mais importantes para cada uma das instituições entrevistadas.
\end{abstract}

\section{Palavras-Chave}

Alfabetização computacional, Tecnologia da Informação, Inclusão Digital.

\begin{abstract}
From time to time, it has been seen increased the dissemination of computing devices in the society. However, recent research shows situations of individuals equipped with IT devices, but technologically unprepared. Based on this context, it is evident that there is a minimal learning about computational aspects, which assigns the term Computer Literacy (CL). The objective of this study is to evaluate the relevance of CL aspects in the knowledge base formation of young Brazilians. This work is characterized by being an exploratory research using the in-depth interview technique to capture perceptions of three Brazilian institutions related to the subject of study. The research confirms the relevance of CL's knowledge and skills, identifying particulars of the most important aspects for each of the surveyed institutions.
\end{abstract}

\section{Categories and Subject Descriptors}

Permission to make digital or hard copies of all or part of this work for personal or classroom use is granted without fee provided that copies are not made or distributed for profit or commercial advantage and that copies bear this notice and the full citation on the first page. To copy otherwise, or republish, to post on servers or to redistribute to lists, requires prior specific permission and/or a fee.

SBSI 2016, May 17-20, 2016, Florianópolis, Santa Catarina, Brazil.

Copyright SBC 2016
K.3.2 [Computers and Education]: Computer and Information Science Education - Literacy.

\section{General Terms}

Human Factors.

\section{Keywords}

Computer literacy, Information Technology, Digital inclusion.

\section{INTRODUÇÃO}

$\mathrm{O}$ uso cotidiano dos computadores sobretudo o acesso à internet tem se tornado bastante comum no mundo todo. Empresas e órgãos de governo de diversos países estão cada vez mais dependentes de sistemas de Tecnologia de Informação (TI). A adoção dos elementos de TI influencia diversos aspectos de vida na sociedade atual $[8,18]$ propiciando comodidades para: pagamento de contas, comunicação, pesquisa de informações, compra de produtos, acesso a vídeos/música e muito mais [4].

A influência da TI no processo de aprendizagem também é grande. O método tradicional praticado por décadas, baseado em papel, lápis, livros, não pode manter-se alheio a existência da internet, dado o enorme volume de informações úteis para o ensino de todas as áreas de conhecimento e dos diversos níveis [8].

Entretanto, se por um lado há uma parcela da sociedade que usufrui de benefícios e conforto oriundos da evolução digital, criando novos caminhos para o estabelecimento de relacionamentos, construção de conhecimento, colaboração e inovações, há uma parcela considerável da população que se encontra ainda excluída deste universo [2].

Como um fator atenuante, ao longo do tempo presenciamos a redução gradativa dos custos dos dispositivos computacionais, possibilitando que estes estejam cada vez mais presentes nos ambientes familiares [7]. Somado a este aspecto, a indústria da tecnologia dedica-se a criar dispositivos e aplicativos com a usabilidade mais natural possível, ampliando os índices de autoaprendizagem $[5,9]$.

Há um mito de que os conhecimentos e habilidades básicas sobre os dispositivos de TI são incorporadas naturalmente pelas gerações mais recentes, em especial dos provenientes de classes sociais mais privilegiadas, uma vez que crescem rodeado de tecnologia em seu cotidiano [10]. Entretanto, pesquisas apontam que o auto aprendizado e o aprendizado proveniente de pessoas próximas restringe-se a uma abrangência insuficiente, usualmente focados nas ferramentas de comunicação [11], jogos eletrônicos [8] ou acessam a internet apenas como navegadores passivos [4]. 
As exigências das habilidades computacionais dos alunos de graduação tornaram-se necessárias em todos os cursos e de todas as áreas de conhecimento [9]. As empresas privadas e governamentais também estão em busca constante de informatização, devido a contribuição da tecnologia na redução de custo e aumento de produtividade. Por decorrência, também se aumentou a exigência de conhecimento das habilidades computacionais nos processos seletivos para ingresso no mercado de trabalho, mesmo para as atribuições profissionais mais simples [5].

Dado este contexto, evidencia-se que há um conjunto mínimo de conhecimento e habilidades digitais, os quais capacitam os indivíduos à produção ativa como alunos nas instituições de ensino de graduação, ampliam as oportunidades de ingresso no mercado de trabalho, e também incorporam melhorias na vida cotidiana. Para este conjunto mínimo de conhecimento e habilidades digitais atribui-se o termo Alfabetização Computacional (AC).

O objetivo deste estudo é avaliar a relevância dos conhecimentos e habilidades da AC no contexto das instituições de ensino médio, ensino técnico e das organizações brasileiras. A pesquisa parte de um referencial teórico sobre conhecimentos e habilidades de AC considerados relevantes para a vida pessoal e profissional de alunos de ensino médio e se utiliza de entrevistas em profundidade para avaliar a relevância desses conhecimentos e habilidades no contexto brasileiro.

Nas próximas seções deste artigo serão descritos: a fundamentação teórica, os procedimentos metodológicos utilizados, a aplicação das entrevistas em profundidade, os resultados obtidos e as conclusões finais do trabalho.

\section{FUNDAMENTAÇÃO TEÓRICA}

O conceito convencional de alfabetização, até meados dos anos 70, era associado à instrução formal oferecida pelas escolas [12], mais especificamente relacionado a habilidade de leitura e escrita. Particularmente aos adultos que não tinham acesso a este processo de aprendizado, atribuía-se a denominação de analfabetos.

A disseminação dos elementos de TI determinou uma mudança no processo de comunicação, sobretudo no conceito de alfabetização. Esta nova alfabetização representa um conjunto de conhecimentos necessários para que um indivíduo esteja inserido em seu contexto social, habilitado a comunicar-se por meio das ferramentas existentes $[7,12]$.

Este conjunto de conhecimentos e habilidades sobre AC correspondem a um aprendizado universal, necessário para todas as pessoas [23], em especial por possibilitar o desenvolvimento de novos modelos cognitivos, incentivando a inovação e a criatividade por meio de novos pensamentos e hipóteses [3]. A questão remanescente seria definir quais e qual profundidade de conhecimento computacional cada indivíduo precisaria desenvolver [23].

Mason e Morrow [15] relatam que a partir da metade de 1980, o foco da AC foi direcionado aos pacotes de software. A abrangência de conhecimentos da AC se traduzia na habilidade de uso de processadores de texto, planilhas, apresentações gráficas e gerenciamento básico de arquivos. Nesse período os cursos introdutórios de AC despertavam interesse pelas mais diversas áreas de conhecimento, não se restringindo aos alunos da área de ciências da computação.

Diversos autores [3, 6, 10, 13, 15] sinalizam não haver um consenso sobre as nomenclaturas e abrangências adotadas para classificar os estágios de aprendizado computacional. Painéis de discussão envolvendo especialistas de diversas universidades se reuniram para debater sobre qual termo deveria ser adotado para definir o primeiro estágio de conhecimento e habilidades computacionais [20].

Assim como a natureza do termo alfabetização, a AC deve corresponder primariamente a um conjunto mínimo de conhecimentos e habilidades, o qual possibilite maior amplitude possível de aprendizagem para a sociedade $[1,5]$.

Mason e Morrow [15] destacam que a formação adequada da aprendizagem sobre AC demanda um equilíbrio necessário sobre dois elementos: (1) conhecimento, o qual corresponde à conscientização e ampliação da capacidade cognitiva de caráter instrutivo, como ética e riscos; e também (2) habilidades, correspondente ao desenvolvimento de capacitações de uso prático, como por exemplo editores de texto e ferramenta de busca.

Constata-se que a AC é um tema dinâmico, necessitando acompanhar a curva acentuada da evolução da tecnologia na sociedade, criando constantemente a necessidade de revisão [6]. Goldweber, Barr e Leska [6] propõem que a AC seja predominantemente conceitual, possibilitando maior independência do aspecto evolutivo dos softwares [6]. Associar AC ao conhecimento de uso baseado em suítes de um único fabricante, como Microsoft ${ }^{\mathrm{MR}}$ por exemplo, seria um equívoco, uma vez que o aprendizado resultante seria restrito e limitado $[10,14]$

Myers et al. [17] propõem que o conhecimento necessário para a AC deve corresponder ao desenvolvimento de um pensamento crítico da tecnologia no uso de diversos dispositivos, softwares e recursos conectados. Considerando a velocidade da evolução tecnológica, seria necessário criar um aprendizado autônomo para resolver os problemas por meio da tecnologia disponível à época, ou seja, que possibilite "aprender como aprender".

$\mathrm{O}$ advento da internet ocasionou profundas alterações no significado do termo AC, considerando necessário desenvolver um entendimento criterioso sobre os meios de comunicação e as informações publicadas [11]. Os autores observam que a conectividade com a rede mundial tem se tornado cada vez mais natural, de modo que a AC precisaria incorporar também a habilidade de avaliar informações publicadas na internet.

$\mathrm{O}$ entendimento sobre AC segundo Turk [21], representaria o essencial e universal para toda a sociedade, com foco no entendimento das implicações legais de atos ilícitos no mundo digital, assim como também a importância da conscientização sobre os riscos de tornar-se vítimas de cybercrimes.

\section{METODOLOGIA DA PESQUISA}

Este trabalho caracteriza-se por ser uma pesquisa exploratória [19] com o objetivo de avaliar a relevância dos conhecimentos e habilidades da AC nos contextos das instituições de ensino médio, de ensino técnico e das organizações brasileiras. A pesquisa é composta por quatro fases. $\mathrm{Na}$ primeira fase foi realizada uma revisão da literatura que identificou conhecimentos e habilidades relacionados à $\mathrm{AC}$, voltados para alunos do ensino médio.

$\mathrm{Na}$ segunda fase foi elaborado um instrumento com base nos conhecimentos e habilidades relacionados à $\mathrm{AC}$ levantados na literatura e fundamentados no modelo de referência baseado na técnica Delphi. Segundo Linstone e Turoff [24], cenários de pesquisa que possuem um problema que não é solucionado por meio de técnicas analíticas precisas, e cujo grupo de indivíduos com capacitação para analisar esse problema são oriundas de formações distintas em relação a experiência ou especialização, são adequados 
para o uso da técnica Delphi. Na terceira fase foi aplicado o instrumento aos especialistas e analisados os resultados, e na quarta e última fase foram elaboradas as considerações finais da pesquisa.

Os procedimentos metodológicos estão descritos a seguir em três tópicos: modelo de referência da pesquisa; unidades de análise; procedimentos de coleta de dados; e limitações da pesquisa.

\subsection{Modelo de Referência da Pesquisa}

$\mathrm{O}$ modelo de referência desta pesquisa se baseia nos conceitos apresentados na revisão da literatura e no trabalho de Wang e Prado [22], que identificaram 15 aspectos mais relevantes da $\mathrm{AC}$, por meio da aplicação da técnica Delphi em 22 especialistas sobre o tema. Esses aspectos estão apresentados na Tabela 1.

Tabela 1. Aspectos mais relevantes da AC

\begin{tabular}{|l|l|l|}
\hline Dimensão da AC & Aspectos da AC & Mnemônico \\
\hline \multirow{3}{*}{ Ética e Legalidade } & Ética & E1 \\
\cline { 2 - 3 } & Privacidade & E2 \\
\hline Interatividade & Ferramentas de busca & I1 \\
\cline { 2 - 3 } & E-mail & $\mathrm{I} 2$ \\
\cline { 2 - 3 } & Critérios de busca & $\mathrm{I} 3$ \\
\cline { 2 - 3 } & Mensagem instantânea & $\mathrm{I} 4$ \\
\hline Riscos e Impactos & Segurança & $\mathrm{R} 1$ \\
\cline { 2 - 3 } & Vírus & $\mathrm{R} 2$ \\
\cline { 2 - 3 } & Boas Práticas & $\mathrm{R} 3$ \\
\hline \multirow{3}{*}{ Perramentas de } & Editor de Texto & $\mathrm{F} 1$ \\
\cline { 2 - 3 } & Planilhas eletrônicas & $\mathrm{F} 2$ \\
\cline { 2 - 3 } & Apresentação & $\mathrm{F} 3$ \\
\cline { 2 - 3 } & Sistema Operacional & $\mathrm{F} 4$ \\
\hline Padrões digitais & Padrões de Documentos & $\mathrm{P} 1$ \\
\cline { 2 - 3 } & Protocolos & $\mathrm{P} 2$ \\
\hline
\end{tabular}

A seguir uma descrição dos 15 aspectos destacados por Wang e Prado [21]:

(E1) Ética: conhecimento sobre o limite das ações digitais de cada indivíduo de modo a não causar dano de qualquer natureza a outros indivíduos;

(E2) Privacidade: conhecimento sobre o controle da exposição e disponibilidade das informações de cada indivíduo, bem como a não violação da privacidade da informação digital de outros indivíduos, como acesso ou publicação não autorizada;

(I1) Ferramentas de busca: habilidade para uso de sites disponibilizados na internet para encontrar informações de interesse publicado em algum outro site na internet;

(I2) E-mail: habilidade para composição, envio e recebimento de mensagens eletrônicas por meio de correio eletrônico;

(I3) Critérios de busca: conhecimento para o discernimento sobre a fidedignidade, veracidade e completude das informações publicadas na internet, uma vez que a internet é um ambiente livre de publicação;

(I4) Mensagens instantâneas: habilidade para troca de mensagens curtas em tempo real, conhecido também como Chat, tanto para computadores como também para celulares móveis;

(R1) Segurança: conhecimento sobre o conjunto de boas práticas para a autoproteção individual, tanto em ambiente doméstico quanto em ambiente profissional, como política de definição de senhas, restrições para divulgação de seus dados pessoais, cópia de segurança, entre outros;

(R2) Vírus: conhecimento sobre existência de vírus eletrônicos, os impactos e quais as boas práticas que precisam ser adotadas para evitar o contágio dos dispositivos pessoais;

(R3) Boas Práticas: conhecimento sobre os malefícios decorrentes do excesso de uso e forma de descarte mais adequada dos dispositivos de TI em obsolescência ou defeituosos;

(F1) Editor de Texto: habilidade para uso de aplicativo para criação e edição de arquivos de texto plano;

(F2) Planilhas eletrônicas: habilidade para uso de aplicativo que disponibiliza tabelas para organização de dados, realização de cálculos e apresentação de consolidações de informação;

(F3) Apresentação: habilidade para uso de aplicativo para elaboração de apresentações por meio da composição de um conjunto de slides, os quais podem conter desenhos, imagens, texto, animações e vídeo;

(F4) Sistema Operacional: habilidade para uso do software básico que disponibiliza um ambiente de suporte aos usuários para a execução de aplicativos e organização das informações, por meio da representação de pastas e arquivos;

(P1) Padrões de Documentos: habilidade para identificar e converter documentos em formatos padronizados para divulgação segura e simplificada. Formatos padronizados de documentos possibilitam o acesso ao seu conteúdo sem necessidade de aplicativos específicos. Por exemplo, a conversão de documentos, planilhas, apresentações em PDF (Portable Document Format);

(P2) Protocolos: conhecimento sobre a identificação básica do protocolo de comunicação de internet ativo, em especial para reconhecer conexões seguras (HTTP ou HTTPs) para digitação de dados sigilosos, como senhas por exemplo.

\subsection{Unidades de Análise e Proposições da Pesquisa}

Foram selecionadas três unidades de análise, cada qual com vínculo diferenciado e complementar em relação ao público alvo deste estudo: jovens na faixa etária correspondente ao ensino médio. Segue a caracterização destas unidades de análise.

\subsubsection{Formação de Docentes do Ensino Médio (FD)}

Esta unidade de análise constitui uma instituição de capacitação de docentes de ensino médio e coordenadores pedagógico sobre a incorporação da tecnologia na prática cotidiana das aulas. Oferece serviços como: desenvolvimento de processos de ensino e apoio à preparação de material didático adequado. Essa unidade de análise atua indiretamente na capacitação de $\mathrm{AC}$ dos alunos de ensino médio, mas possui a missão de contribuir para o desenvolvimento holístico dos alunos, no aprendizado dos conhecimentos necessários e dotá-los da capacidade de autodesenvolvimento.

Considerando-se as características apresentadas desta unidade de análise, adotaram-se a seguintes proposições de pesquisa:

(P1) Dimensões de AC. Sendo uma instituição de capacitação de docentes e coordenadores pedagógico e tendo a missão contribuir com o aprendizado, as dimensões de AC mais enfatizadas devem ser: Ética e Legalidade; Interatividade; e Riscos e Impactos. 
(P2) Aspectos de AC. Sendo uma instituição de capacitação de docentes e coordenadores pedagógico e tendo a missão de contribuir com o aprendizado, os aspectos de AC de maior relevância são aqueles relacionados à ampliação da aprendizagem.

\subsubsection{Escola Técnica (ET)}

Esta unidade de análise constitui uma Escola Técnica Estadual de São Paulo (ETEC), situada na região da zona leste da cidade. A missão dessas escolas técnicas é a preparação dos alunos com as habilidades práticas mais demandadas pelo mercado de trabalho, preparando-os para a conquista do primeiro emprego ou para a recolocação. Esta instituição ministra cursos técnicos integrados ao ensino médio oferecendo três especialidades: Comunicação Visual, Modelagem do Vestuário e Química.

Considerando-se as características apresentadas desta unidade de análise, adotaram-se as seguintes proposições de pesquisa:

(P3) Dimensões de AC. Sendo uma instituição que tem como missão contribuir com o aprendizado é a preparação dos alunos com as habilidades práticas mais demandadas pelo mercado de trabalho, as dimensões de AC mais enfatizadas devem ser: Interatividade; Ferramentas de Produtividade; e Padrões Digitais.

(P4) Aspectos de AC. Sendo uma instituição que tem como missão contribuir com o aprendizado é a preparação dos alunos com as habilidades práticas mais demandadas pelo mercado de trabalho, os aspectos de AC de maior relevância são aqueles relacionados à ampliação de aprendizagem e à produtividade.

\subsubsection{Empresa $(E)$}

Esta unidade de análise constitui uma corporação privada que contrata frequentemente jovens para compor o quadro de colaboradores. Trata-se de uma associação de concessionárias de rodovias formada por 59 empresas privadas distribuídas em doze estados brasileiros. Atua na defesa dos interesses das associadas e no suporte ao aperfeiçoamento do desempenho das empresas. Ao contratar jovens em seu primeiro emprego, esta unidade de análise considera necessário coloca-los em contato com uma diversidade de atribuições profissionais e, consequentemente, com diversos elementos de TI.

Considerando-se as características apresentadas desta unidade de análise, adotou-se a seguinte proposição:

(P5) Dimensões de AC. Sendo uma instituição que contrata jovens que serão colocados em contato com uma diversidade de atribuições profissionais, as dimensões de $\mathrm{AC}$ mais enfatizadas devem ser: Ética e Legalidade; Interatividade; e Ferramentas de Produtividade.

(P6) Aspectos de AC. Sendo uma instituição que contrata jovens que serão colocados em contato com uma diversidade de atribuições profissionais, os aspectos de AC de maior relevância são aqueles relacionados às necessidades práticas da atuação no mercado de trabalho.

\subsection{Procedimentos e Coleta de Dados}

Para atender ao objetivo desta pesquisa de avaliar a relevância dos conhecimentos e habilidades da $\mathrm{AC}$ nos contextos das instituições de ensino médio ou ensino técnico e das organizações brasileiras, foi selecionado o método de entrevista em profundidade.

Devido ao seu carácter exploratório este tipo de entrevista é especialmente útil na investigação de temas onde existe pouco conhecimento sobre o mesmo. Apesar desse método ter um roteiro para a entrevista, este não é fechado e o entrevistador pode adaptálo de acordo com o desenvolvimento da entrevista. Segundo Mattos [16] a entrevista em profundidade tem sido cada vez mais utilizada em trabalhos de pesquisa.

A aplicação da entrevista em profundidade foi baseada no instrumento de pesquisa baseado nos aspectos de AC extraídos da literatura. A aplicação das entrevistas ocorreu durante o mês de julho de 2015.

Para cada uma das unidades de análise selecionadas, foi identificado um representante para as entrevistas. Foi constatado que papel desses representantes nas respectivas instituições têm envolvimento direto com o objeto de estudo, de modo que a opinião dos entrevistados sobre o tema se aproxima da opinião da unidade de análise.

O procedimento de coleta de dados se iniciou com o envio do instrumento de pesquisa por correio eletrônico, acompanhado de uma explicação e de uma contextualização. Estabeleceu-se com cada entrevistado um procedimento de análise e preenchimento prévio do instrumento de pesquisa. Em seguida, o instrumento preenchido foi analisado pelos pesquisadores e agendada uma entrevista pessoal para explorar o conteúdo com maior profundidade.

A participação de cada entrevistado foi anônima, entretanto as características de cada um estão descritas a seguir, seguido da transcrição das respectivas entrevistas.

\subsection{Limitações da Pesquisa}

Convém tratar com parcimônia os resultados obtidos neste estudo, devido às limitações decorrentes da natureza da pesquisa, em especial as relacionadas ao método de pesquisa. Apenas uma pessoa foi entrevistada por unidade de análise e mesmo sendo selecionado alguém de forma criteriosa e com visão ampla da unidade, as opiniões podem não refletir o posicionamento da unidade em relação a AC. Além disso, os resultados foram baseados nas opiniões das entrevistas em profundidade conduzidas pelos pesquisadores e apresentam subjetividade na interpretação.

Trata-se de um estudo exploratório que visa ampliar o conhecimento sobre a importância da AC nas instituições brasileiras sem a pretensão de generalizar os resultados para as demais instituições brasileiras. Adicionalmente, os aspectos relevantes da $\mathrm{AC}$ analisados neste estudo representam a realidade tecnológica da década de 2010, sendo sujeito a mudanças futuras. Entretanto, apesar dessas limitações, os resultados obtidos são satisfatórios em relação aos objetivos propostos.

\section{APLICAÇÃO DAS ENTREVISTAS EM PROFUNDIDADE}

Os 15 aspectos de AC considerados de absoluta importância, apresentados no modelo de referência desta pesquisa foram submetidos à três unidades de análise distintas. Para cada uma das unidades analisadas, buscou-se identificar o subconjunto de cinco aspectos mais relevantes e de cinco aspectos menos relevantes, além de verificar a existência de algum aspecto da $\mathrm{AC}$ não descrito pelo modelo de referência.

Todos os 15 aspectos apresentados foram considerados importantes pelos entrevistados das três unidades de análise e nenhum novo aspecto foi identificado. O subconjunto de cinco aspectos da AC classificados como relevantes, por unidade de análise, estão apresentados na Tabela 2: 
Tabela 2. Aspectos relevantes da AC por unidade de análise

\begin{tabular}{|c|c|c|c|c|}
\hline $\begin{array}{l}\text { Dimensão da } \\
\text { AC }\end{array}$ & Aspecto & FD & ET & $\mathbf{E}$ \\
\hline \multirow{2}{*}{$\begin{array}{l}\text { Ética e } \\
\text { Legalidade }\end{array}$} & E1 & \multirow[t]{2}{*}{5} & \multirow[t]{2}{*}{3} & \\
\hline & E2 & & & \\
\hline \multirow[t]{4}{*}{ Interatividade } & $\mathrm{I} 1$ & 1 & \multirow{4}{*}{1} & 2 \\
\hline & $\mathrm{I} 2$ & 3 & & 5 \\
\hline & $\mathrm{I} 3$ & 2 & & \\
\hline & I4 & 4 & & 3 \\
\hline \multirow{3}{*}{$\begin{array}{l}\text { Riscos e } \\
\text { Impactos }\end{array}$} & R1 & & \multirow[t]{3}{*}{5} & \multirow{3}{*}{4} \\
\hline & $\mathrm{R} 2$ & & & \\
\hline & R3 & & & \\
\hline \multirow{4}{*}{$\begin{array}{l}\text { Ferramentas de } \\
\text { Produtividade }\end{array}$} & F1 & & \multirow{4}{*}{$\begin{array}{l}4 \\
2\end{array}$} & \multirow{6}{*}{1} \\
\hline & $\mathrm{F} 2$ & & & \\
\hline & F3 & & & \\
\hline & $\mathrm{F} 4$ & & & \\
\hline \multirow[t]{2}{*}{ Padrões digitais } & P1 & & & \\
\hline & $\mathrm{P} 2$ & & & \\
\hline
\end{tabular}

\subsection{Unidade de Análise I - Formação Docente}

A pessoa entrevistada é sócio-diretor da unidade de análise e ministra aulas no ensino superior em cursos de pedagogia. Tem nível de mestrado em mídias interativas, pós-graduação em design de multimídia, gestão em educação à distância e graduação em pedagogia. Desenvolve pesquisa em: mídias digitais, tecnologias digitais na educação e mídia-educação.

Como justificativa de escolha destes cinco aspectos da AC, a pessoa entrevistada destacou a grande importância da internet como fonte de informação e aprendizagem. Nessa linha de raciocínio os dois primeiros aspectos - Ferramentas de Busca e Critérios de Busca correspondem a duas habilidades que, se somadas com a curiosidade dos jovens, possibilitariam descobrir informações novas e agregar conhecimento com autonomia. Os dois aspectos seguintes - E-mail e Mensagens Instantâneas - representam, segundo o entrevistado, a importância da habilidade de se comunicar na rede digital. Estes aspectos também podem contribuir com a autoaprendizagem. E, por fim, o aspecto Ética fator necessário para uma convivência adequada, alinhada às normas de legislação no meio digital, e em concordância com os valores da sociedade digital.

Aspectos da AC classificados como de menor importância pelo entrevistado, sem ordenação por grau de importância, foram: Planilhas Eletrônicas, Sistema Operacional, Protocolos, Vírus e Segurança. Como justificativa da escolha dos cinco aspectos da AC de menor relevância, segundo o entrevistado, seriam os aspectos menos demandados no cotidiano dos jovens. Entre esses destacamse por exemplo, as "planilhas" e os "protocolos". O entrevistado também destacou a baixa importância do conhecimento de uso do aspecto Sistema Operacional por não corresponder a um objetivo fim, mas sim um mero meio para organizar, executar e acessar aplicativos ou informações de real interesse. Segundo o entrevistado, os aspectos Vírus e Segurança possuem relevância reconhecida, mas ele acredita que grande parte dos aplicativos tendem a migrar para a internet, com acesso on-line. Dessa forma, além de minimizar a incidência de vírus, as políticas de segurança serão automatizadas por procedimentos. Isso reduz a preocupação dos usuários com a necessidade de ações, tais como, troca de senha periódica e análise do grau de segurança das senhas, entre outras.

Como consideração final, o entrevistado destacou duas grandes fragilidades do processo de ensino da $\mathrm{AC}$ no ensino médio, em especial o público. A primeira delas é a infraestrutura precária, mais especificamente em relação a conectividade, resultando em um acesso à internet de qualidade muito baixa, dificultando o desenvolvimento adequado de programas de ensino. A segunda é a formação inadequada de conhecimentos da AC dos professores, pois eles são um dos principais agentes potenciais de transformação da qualidade do ensino, os quais precisam ser motivados e instrumentados adequadamente.

\subsection{Unidade de Análise II - Escola Técnica}

A pessoa entrevistada foi coordenador dos cursos técnicos de informática e internet no período de 2010 a 2012 na ETEC, atuando na elaboração dos projetos pedagógicos e didáticos da mesma.

Como justificativa de escolha destes cinco aspectos da $\mathrm{AC}$, selecionados como importantes pelo entrevistado, figuram os três aspectos considerados relevantes para o uso seguro, proveitoso e eficaz da internet: Critérios de Busca, Ética e Segurança. E os dois aplicativos da relação - Editor de Texto e Planilhas Eletrônicas foram considerados conhecimentos relevantes para uso cotidiano, em especial para o ingresso nas empresas.

Aspectos da AC classificados como de menor importância pelo entrevistado, sem ordenação por grau de importância, foram: Apresentação, Padrões de Documentos, E-mail, Mensagens Instantâneas e Ferramentas de Busca.

$\mathrm{O}$ argumento apresentado pelo entrevistado para a classificação destes aspectos com baixa importância corresponde a duas motivações: baixa demanda do mercado (aspecto Apresentação); e aspectos usuais ou de fácil aprendizado pelo próprio aluno ou com colegas (aspectos Ferramentas de Busca, Padrões de Documentos, E-mail e Mensagens Instantâneas). Segundo o entrevistado, considerando a necessidade elaborar um conteúdo programático de AC para a grade curricular da instituição, baseado em uma quantidade limitada de aspectos, o critério a ser adotado seria: alta importância para o que o mercado de trabalho demanda, e baixa importância para o que não é usual no mercado e para aquilo que o aluno conseguiria aprender por si.

Como considerações finais, o entrevistado destacou as iniciativas de se promover o aumento da aprendizagem da AC por meio de interações constantes dos alunos com representante do mercado de trabalho. E assim, por meio de demonstrações das demandas do cotidiano do mercado de trabalho, espera-se motivar os jovens no aprendizado geral, sobretudo o relacionado à tecnologia.

\subsection{Unidade de Análise III - Empresa}

A pessoa entrevistada tem formação e mestrado em administração, bacharel em direito. Atuou como diretor administrativo-financeiro na Prefeitura Municipal de São Paulo, foi gerente administrativo e de projetos, e gerente de $\mathrm{RH}$. Atualmente, é professor assistente e diretor executivo da ABCR (Associação Brasileira de Concessionárias e Rodovias).

Como justificativa de escolha destes cinco aspectos da $\mathrm{AC}$, o entrevistado justificou que foram selecionados os conhecimentos e habilidades relacionados ao uso adequado e otimizado dos dispositivos computacionais. Segundo ele, o desenvolvimento de habilidades que favoreçam a facilidade de uso e compreensão da capacidade oferecida pelos dispositivos computacionais é mais relevante que o objetivo do uso em si, e facilita o aprendizado de uso de qualquer aplicativo. Por essa razão o aspecto Sistema Operacional foi considerado o mais relevante. Outras justificativas também foram acrescentadas: a ferramenta de busca corresponde a um canal para um auto aprendizado e esclarecimento de dúvidas; e 
a consciência sobre os vírus é necessária para evitar infecções digitais nos equipamentos das empresas e a habilidade de uso de ferramentas de comunicação é demandada no cotidiano profissional.

Aspectos da AC classificados como de menor importância pelo entrevistado, sem ordenação por grau de importância, foram: Critérios de Busca, Privacidade, Ética, Boas Práticas e Segurança.

Como justificativa da escolha dos cinco aspectos da AC de menor relevância, segundo o entrevistado, seriam os aspectos relacionados a valores e aspectos secundários no uso dos dispositivos computacionais. Apesar dessa escolha, o entrevistado destaca reconhecer a relevância da ética e da moral no comportamento de qualquer indivíduo, independentemente da relação com o uso de computadores, e por esse motivo não teriam relação direta com as habilidades e capacitações relacionadas diretamente à AC.

As considerações finais do entrevistado sinalizam a importância do incentivo à disseminação ampla sobre os conhecimentos da AC pelas empresas, favorecendo inclusive o acesso permanente dos jovens às tecnologias de mais difícil acesso. Segundo ele, dessa forma, desenvolvem-se a criatividade e a inovação a partir da aplicação de dispositivos computacionais no cotidiano profissional.

\section{ANÁLISE DOS RESULTADOS}

Nesta seção foram avaliados os dados coletados nas entrevistas em profundidade e as proposições inicialmente formuladas para cada uma das unidades de análise. Em seguida as constatações destacadas foram analisadas.

Primeiro é importante destacar que as três unidades de análise consideraram que os 15 aspectos da $\mathrm{AC}$ são todos importantes. Porém, os objetivos desta pesquisa direcionaram as entrevistas em profundidade para a escolha de subconjuntos de maior e menor importância, com o objetivo de identificar as particularidades da AC nos distintos contextos de cada unidade de análise. A avaliação comparativa dos resultados das três entrevistas evidenciou a distinção de opiniões sobre a relevância dos principais aspectos da AC para cada uma das unidades de análise, possivelmente devido às distintas experiências de suas respectivas atuações profissionais. Convém também registrar que nenhuma das três unidades de análise notou a ausência de algum aspecto relevante da $\mathrm{AC}$, em relação à listagem apresentada no instrumento de pesquisa. Isso constitui um indício da atualidade dos aspectos de AC destacados por Wang e Prado [21].

A análise dos resultados está apresentada a seguir, por proposição de pesquisa.

\subsection{Proposição P1}

Na proposição $\mathrm{P} 1$ considerou-se que a instituição de capacitação de docentes e coordenadores pedagógicos indicaria maior relevância nas dimensões relacionadas ao aprendizado (Ética e Legalidade; Interatividade; e Riscos e Impactos).

Todos os quatros aspectos correspondentes à dimensão de $\mathrm{AC}$ interatividade foram selecionados pela instituição Formação Docente, corroborando com a destacada importância da interatividade mencionada na proposição $\mathrm{P} 1$.

A quinta escolha desta instituição foi a Ética, também confirmando a proposição $\mathrm{P} 1$.

Entretanto, diferentemente da expectativa apontada na proposição P1, os aspectos Segurança e Vírus, correspondentes à dimensão de Riscos e impactos, foram classificados como menor importância pela instituição Formação Docente. Inclusive Turk [21] menciona a grande relevância da autoproteção no mundo digital, devido aos riscos de prejuízos reais decorrentes dos cybercrimes. Porém, convém ponderar a análise de Turk [21] considerando a dinâmica da evolução tecnológica. Temos presenciado mais recentemente a expansão da tecnologia computação em nuvem, em tem se tornado cada vez maior a oferta de uso aplicativos como serviço, acessandoos diretamente na internet. Desta forma as políticas de segurança destes aplicativos na nuvem passa a ser gerenciada por corporações profissionais, possivelmente com equipe técnica capacitada em aspectos de segurança.

\subsection{Proposição P2}

A proposição P2 considera a expectativa da unidade Formação Docente em classificar maior importância nos aspectos relacionados com a ampliação da aprendizagem

O conceito de ampliação da aprendizagem possivelmente esteja relacionado com o acesso a bases de informação, e o acesso à comunicação com a comunidade, o qual possibilita a troca de experiências com outros indivíduos. Somado a isso, não basta ter acesso, é fundamental desenvolver critérios para o discernimento da fidedignidade das informações. Portanto, a ampliação da aprendizagem possivelmente é favorecida pelos aspectos de AC relacionados à interatividade.

Observa-se que a unidade de análise Formação Docente classificou com alta importância todos os quatro aspectos relacionados a dimensão de AC Interatividade, confirmando fortemente a proposição P2.

\subsection{Proposição P3}

A proposição P3 relaciona-se com a missão das unidades Escolas Técnicas, estar orientado à preparação dos alunos para o mercado de trabalho, tendendo a privilegiar as dimensões de $\mathrm{AC}$ relacionadas à Interatividade, Ferramentas de Produtividade e Padrões Digitais.

Em relação a dimensão de AC Interatividade, a unidade Escola Técnica selecionou apenas um aspecto (critérios de busca) como relevante e os demais três aspectos (ferramentas de busca, $e$-mail e mensagens instantâneas) como pouco relevantes, com a justificativa de fácil aprendizado, e não de pouca importância para AC em geral. Complementando esta análise de fato encontra-se na literatura pesquisada, referências de que a autoaprendizagem favorece a assimilação de uso das ferramentas de comunicação [11]. Considera-se, portanto, possivelmente a menor necessidade de aprendizagem destes em ambiente escolar, possibilitando a confirmação da Interatividade como importante para esta unidade.

Em relação a dimensão de AC Ferramentas de Produtividade, dois aspectos foram considerados relevantes (editor de texto e planilhas eletrônicas) e apenas um considerado pouco relevante (apresentação). Possibilita-se também confirmar a dimensão de AC Produtividade como importante, sendo que na visão desta unidade, o aspecto "apresentação" seria menos demandado pelo mercado.

$\mathrm{O}$ aspecto Padrões de Documentos, correspondente à dimensão de AC Padrões digitais recebeu classificação de baixa relevância, diferentemente da expectativa. Convém possivelmente rever futuramente este ponto da proposição P3 em um estudo mais aprofundado. 


\subsection{Proposição P4}

A proposição P4 considera a expectativa de atribuição de importância de aspectos relacionados à ampliação de aprendizagem e produtividade pela unidade Escola Técnica.

Considera-se a confirmação de importância referente à ampliação da aprendizagem pela entrevista desta unidade, dado a relação deste conceito em relação a Interatividade, mencionado em P2, e a relevância atribuída à esta dimensão de $\mathrm{AC}$, conforme justificado em P3.

O entendimento do conceito produtividade possivelmente esteja relacionado às ferramentas de produtividade. Neste contexto, a unidade Escola Técnica indica uma visão parcialmente alinhada, uma vez que apenas dois dos quatro aspectos de produtividade, segundo esta unidade, é relevante. Não foi encontrado referência de que os aspectos de Apresentação e Sistemas Operacionais sejam assimilados pela autoaprendizagem.

\subsection{Proposição P5}

A proposição P5 considera que as empresas enfatizam na seleção de candidatos a importância das dimensões AC Ética e Legalidade; Interatividade; e Ferramentas de Produtividade. Estes corresponderiam às características mínimas esperadas de conhecimentos de TIC para uma atuação profissional.

Diferentemente da expectativa, ambos aspectos referentes à Ética e Legalidade foram classificados com baixa importância. A unidade empresa menciona em entrevista que reconhece a importância destas características em profissionais, entretanto não os considera como características de AC, mas sim condutas de comportamento do ser humano em geral. Considera-se conveniente uma exploração futura mais aprofundada destes aspectos, focando uma análise do entendimento das empresas sobre as particularidades de Ética e Legalidade especificamente para o mundo digital.

A dimensão de AC Interatividade esteve bastante presente na classificação de importância da unidade Empresa: três dos quatro aspectos selecionados. Cabe, porém, mencionar que o aspecto de Critérios de Busca além de não ter sido considerando importante pela unidade Empresa foi classificado como pouco relevante no contexto da pesquisa. É possível considerar que no ambiente corporativo a importância de produzir é mais prioritária que a criatividade, em especial para jovens na faixa etária do ensino médio.

Apenas uma ferramenta de produtividade se mostrou presente como importante para a unidade Empresa, com a justificativa que os Sistemas Operacionais constituem base do entendimento dos aplicativos em geral. Confirma-se, portanto, na interpretação da unidade Empresa, a visão de importância da dimensão de AC Ferramentas de Produtividade como um todo.

\subsection{Proposição P6}

Por fim a proposição P6 explora o foco da importância nas necessidades práticas da atuação corporativa. Considerando que nas Empresas a produção é fundamental para o resultado financeiro, portanto espera-se que funcionários, em especial os jovens da idade correspondente ao ensino médio (foco da pesquisa), estejam aptos a produzir com a menor necessidade de treinamento possível.

Nota-se, porém, que pelas classificações da unidade Empresa, espera-se que este jovem potencial de auto evolução. Ao selecionar como importante o aspecto Sistema Operacional, sinaliza-se que a base de conhecimento é necessária, uma vez que apenas este aspecto por si, não possibilita produção alguma. Somado a isso, as ferramentas de comunicação estão fortemente presentes, de modo que a empresa possivelmente considera importante que os jovens se integrem a processo de comunicação para buscar apoio para resolver as demandas apresentadas.

$\mathrm{O}$ aspecto de AC Vírus, também registrado como importante, indica a preocupação das empresas em que os jovens funcionários sejam cuidadosos em não se tornem origem de problemas, consequentemente influenciando negativamente a produtividade.

\section{CONSIDERAÇÕES FINAIS}

Após discorrer sobre o tema AC, fica evidente a complexidade e também a relevância do assunto. Diversos pesquisadores [4, 8, 14 e 17] abordaram o tema ao longo do tempo, possibilitando observar na revisão da literatura o momento que se iniciou a abordagem sobre o assunto, até o momento presente, em que se tornou comum e importante a praticamente todo o mundo, e para todas as classes sociais. Para o período analisado na revisão sistemática, pode-se considerar que é inerente à $\mathrm{AC}$ uma dinâmica acentuada, devido principalmente à necessidade de acompanhar a evolução tecnológicas que se presencia frequentemente. Apesar das mudanças evolutivas, a análise histórica revela um rastro que se preservou ao longo do tempo: os computadores surgiram e se mantêm como importantes aliados no auxílio da resolução dos problemas cotidianos.

A proposição do estudo apresentado ao longo deste trabalho visou descrever a relevância dos conhecimentos e habilidades da AC nos contextos das instituições: ensino médio, ensino técnico e das organizações brasileiras. Foram avaliadas as particularidades da AC identificada por meio da aplicação de entrevistas em profundidade em distintos contextos, a partir de um referencial teórico sobre o tema.

A sociedade atual vivencia um momento em que os computadores estão amplamente disseminados nas mais diversas áreas de atuação, de modo que as aplicações são diversas. Por decorrência, as opiniões sobre quais são os conhecimentos e habilidades relevantes da AC, por vezes, também são controversos dado o resultado coletado nas entrevistas em profundidade.

A partir dos aspectos relevantes identificados neste trabalho é possível observar importantes interpretações sobre a AC atualmente. Destaca-se a alta importância atribuída ao aspecto Ética relacionado à $\mathrm{AC}$. Ou seja, o respeito e moral nas relações digitais é considerado essencial pelos especialistas abordados, a propósito, conforme reforçado pela EMPRESA, a ética é fundamental a todas as relações, sejam reais ou virtuais.

Sugerem-se como trabalhos futuros, a aplicação dos procedimentos metodológicos desta pesquisa em uma amostra probabilística de organizações e instituições de ensino, com o objetivo de ampliar o conhecimento sobre a AC no Brasil e corroborar os resultados aqui apresentados.

\section{REFERENCES}

[1] Bartholomew, K. W. 2004. Computer literacy: is the emperor still exposed after all these years?. J. Comput. Sci. Coll., 323-331.

[2] CGI.BR. 2014. TIC: Domicílios e Empresas 2013. Núcleo de Informação e Coordenação do Ponto BR, São Paulo, 658. 
[3] Cohen, E. 1987. What is computer literacy: the imposter, the sham, and the misdirected. 15th annual conference on Computer Science, (St Louis, Missouri, USA, February 1619), 320-322.

[4] Dednam, E. 2009. Away with computer literacy modules at universities, or not?. Proceedings of the 2009 Annual Conference of the Southern African Computer Lecturers Association - SACLA'09, (Eastern Cape, South Africa, June 29-July 01, 2009) 23-32.

[5] Dyck, V. A., Black, J. P., and Fenton, S. L. 1987. Beyond traditional computer literacy. Proceedings of the eighteenth SIGCSE technical symposium on computer science education - SIGCSE' 87, (St. Louis, USA, February 19-20, 1987), 508-512.

[6] Goldweber, M., Barr, J. and Leska, C. 1994. A new perspective on teaching computer literacy. ACM SIGCSE Bull., 94, 3, 131-135.

[7] Goodman, F. L. 1981. Computers and the future of literacy. Proceedings of the 1981 National Computer Conference AFIPS'81, (Chicago, USA, May 4-7, 1981) 601-604.

[8] Gupta, G. 2006. Computer literacy: essential in today's computer-centric world. ACM SIGCSE Bull., 38, 2, 115-119.

[9] Hartman, J. 1983. Computer literacy objectives for college faculty. in Proceedings of the 11th annual ACM SIGUCCS conference on user services, (New Orleans, USA, October 5-7, 1983), 189-192.

[10] Hoar, R. 2014. Generally Educated In The 21st Century: The Importance Of Computer Literacy In An Undergraduate Curriculum. Proceedings of the Western Canadian Conference on Computer Educucation, (Richmond, Canada, May, 2-3), 5.

[11] Hoffman M. and Vance, D. 2005. Computer literacy: what students know and from whom they learned it. ACM SIGCSE Bull., 356-360.

[12] Lankshear, C. and Knobel, M. 2006. New Literacie: Everyday Practices and Classroom Learning, Second Edition. Open University Press, Benkshire, England, 296.

[13] Leu, D. J., Kinzer, C. K., Coiro, J. L. and Cammack, D. W. 2004. Toward a theory of new literacies emerging from the Internet and other information and communication technologies. Read. Online, 5, 2000, 43-79.
[14] Liao, L., and Pope, J. 2008. Computer literacy for everyone. J. Comput. Sci. Coll., 23, 6, 231-238.

[15] Mason, J. and Morrow, R. 2006. YACLD: yet another computer literacy definition. J. Comput. Sci. Coll., 21, 5, 94100 .

[16] Mattos, P. L. C. L. Análise de entrevistas não estruturadas: da formalização à pragmática da linguagem. In: Silva, A. B.; Godoi, C. K.; Bandeira-De-Melo, R. (orgs). Pesquisa qualitativa em estudos organizacionais: paradigmas, estratégias e métodos. 2. ed. São Paulo, 2010, p. 347-374.

[17] Myers, M., Murray, M., Pérez, J. and Geist, D. 2007. Learner-centered assignments in computer literacy. $J$. Comput. Sci. Coll., 23, 2, 90-96.

[18] Murray, M., and Pérez, J. 2008. Computing for the masses: extending the computer science curriculum with information. J. Comput. Sci. Coll., 24, 2, 220-226.

[19] Selltiz, C.; Wrightsman, L. S.; Cook, S. W. Métodos de pesquisa nas relações sociais. São Paulo: Editora da Universidade de São Paulo, 1987.

[20] Surma, D. R., Geise, M. J., Lehman, J. and Beasley, R. 2012. Computer literacy: what it means and do today's college students need a formal course in it?*. CCSC Midwest. Conf., 28, 1, 142-143.

[21] Turk, J. 2011. Computer literacy as life skills for a web 2.0 world. Proceedings of the 42nd ACM Technical Symposium on Computer Science Education - SIGCSE'11, (Dallas, USA, March 9-12, 2011), 417.

[22] Wang, M. A.; Prado, E. P.V. Aspectos Relevantes da Alfabetização Computacional. Anais da Congresso de Administração, Sociedade e Inovação, 3-4 de dezembro, 2015.

[23] Willoughby, T. 1983. Exposure, knowledge or skill the computer literacy dilemma. Proceedings to the Twentieth Annual Computer Personnel on Research Conference, (Charlottesville, USA, November 17-18, 1983), 75-78.

[24] Linstone, H.; Turoff, M. The Delphi Method Techniques and Applications. 2002 